\title{
Research on ISFLA-Based Optimal Control Strategy for the Coordinated Charging of EV Battery Swap Station
}

\author{
Xueliang Huang, ${ }^{1,2}$ Hao Qiang, ${ }^{1,3}$ Qidong Zhang, ${ }^{1}$ and Haijuan Li \\ ${ }^{1}$ School of Electrical Engineering, Southeast University, Nanjing 210096, China \\ ${ }^{2}$ Jiangsu Key Lab of Smart Grid Technology and Equipment, Zhenjiang 212009, China \\ ${ }^{3}$ School of Information Science and Engineering, Changzhou University, Changzhou 213164, China \\ Correspondence should be addressed to Xueliang Huang; xlhuang@seu.edu.cn
}

Received 28 April 2013; Accepted 24 October 2013

Academic Editor: Yudong Zhang

Copyright (C) 2013 Xueliang Huang et al. This is an open access article distributed under the Creative Commons Attribution License, which permits unrestricted use, distribution, and reproduction in any medium, provided the original work is properly cited.

\begin{abstract}
As an important component of the smart grid, electric vehicles (EVs) could be a good measure against energy shortages and environmental pollution. A main way of energy supply to EVs is to swap battery from the swap station. Based on the characteristics of EV battery swap station, the coordinated charging optimal control strategy is investigated to smooth the load fluctuation. Shuffled frog leaping algorithm (SFLA) is an optimization method inspired by the memetic evolution of a group of frogs when seeking food. An improved shuffled frog leaping algorithm (ISFLA) with the reflecting method to deal with the boundary constraint is proposed to obtain the solution of the optimal control strategy for coordinated charging. Based on the daily load of a certain area, the numerical simulations including the comparison of PSO and ISFLA are carried out and the results show that the presented ISFLA can effectively lower the peak-valley difference and smooth the load profile with the faster convergence rate and higher convergence precision.
\end{abstract}

\section{Introduction}

Over the past decades, many issues, such as energy shortages, serious environmental pollution, and global warming, have increasingly become worldwide concerns. EVs have emerged as a new traffic tool. Compared with internal combustion engine vehicles (ICEVs), which burn fossil fuels, EVs are driven by electricity. They demonstrate considerable advantages in solving the energy crisis and reducing the emissions of carbon dioxide, as well as in providing a means to drastically reduce the man-made pollution. More and more governments, car manufacturers, and energy companies are getting active in development and production of EVs [1].

With the large-scale introduction of EVs, the power grid will face a significant challenge. Many domestic and foreign scholars have carried out researches on the impact of EVs on power distribution system [2-7], which mainly focus on the coordinated charging of EVs but rarely involve the optimal control strategy for the coordinated charging of EV battery swap station. Here, the coordinated charging means that the batteries are controlled to orderly charge for achieving an optimal objective such as minimizing the power losses or maximizing the grid load factor. Tian et al. established a mathematical model of dispatching strategy based on different objective functions which were solved by particle swarm optimization (PSO). The results show that the coordinated charging can lower the peak-valley difference and smooth the load profile [8].

The SFLA is a global optimization algorithm proposed by Eusuff et al. $[9,10]$. It is a memetic metaheuristic that is based on the evolution of memes carried by interactive individuals and a global exchange of information among the frog population. The SFLA draws its formulation from two other search techniques: the local search of the "particle swarm optimization" technique and the competitiveness mixing of information of the "shuffled complex evolution" technique. It locates a global optimum by combining global information exchange and local search, simulating the process of a group of frogs' population-based cooperative seeking food. The strategies of local search and global information exchange make frog leap out of local optimum towards the global optimum solution (food place) with high probability. The algorithm 
has been developed to arrive at near-optimum solutions to complex and large-scale optimization problems which cannot be solved by gradient based mathematical programming techniques [11]. The SFLA has also been applied successfully to solving engineering problems such as traveling salesman problem (TSP) $[12,13]$, unit commitment (UC) problem [14-16], embedding virtual networks [17], speech emotion recognition [18], cognitive radio system [19], and other types of issues.

In this paper, based on the characteristics of the EV battery swap station, the ISFLA-based optimal control strategy for coordinated charging has been investigated. Through the discretization of the solution vector, an ISFLA based on the reflecting method to deal with the boundary constraint is proposed. The comparison of PSO and ISFLA shows that the presented ISFLA can lower the peak-valley difference and smooth the load profile with the faster convergence rate and higher convergence precision.

\section{Shuffled Frog Leaping Algorithm}

As a bioinspired optimization technique, the SFLA is a metaheuristic optimization method, which imitates and models the behavior of frogs searching for food laid on discrete stones randomly located in a pond. In SFLA there is a population, which consists of a set of frogs (solutions). The set of frogs is partitioned into subsets referred to as memeplexes. Different memeplexes are considered as different cultures of frogs, and each memeplex performs a local search. Within each memeplex, the individual frogs hold ideas, which can be influenced by those of other frogs, and evolve through a process of memetic evolution. Frog leaping improves an individual's meme and enhances its performance towards the goal. After a predefined number of memetic evolution steps, ideas are passed among memeplexes in a shuffling process. The local search and the shuffling processes continue until the defined convergence criteria are satisfied.

The SFLA involves a population of possible solutions defined by a set of $p$ randomly generated frogs denoted as $Q=\left[X_{1}, X_{2}, \ldots, X_{p}\right]^{T}$, where $X_{i}=\left(x_{1}, x_{2}, \ldots, x_{i S}\right)$ is the position of the $i$ th frog in the $S$ dimension search space. All the frogs are sorted in a descending order according to their fitness, and the population is divided into $m$ memeplexes, each containing $n$ frogs (i.e., $p=m \times n$ ), in such a way that the first frog goes to the first memeplex, the second frog goes to the second memeplex, the $m$ th frog goes to the $m$ th memeplex, and the $(m+1)$ th frog goes back to the first memeplex, and the process continues in this manner.

In each memeplex the frogs with the best and the worst fitness are represented by $X_{b}$ and $X_{w}$, respectively. The best frog in the whole population is denoted by $X_{g}$. During memeplex evolution, the worst frog $X_{w}$ leaps toward the best frog $X_{b}$, which is formulated as the following updating rule:

$$
\begin{gathered}
D=\operatorname{rand}(0,1) \times\left(X_{b}-X_{w}\right), \\
X_{w_{\text {nnew }}}=X_{w}+D, \quad-D_{\max } \leq D \leq D_{\max },
\end{gathered}
$$

where $D$ denotes the updated step size for frog leaping, rand $(0,1)$ generates a random number between $[0,1]$, and $D_{\max }$ is the maximum distance in one leaping.

If $X_{w_{\text {_new }}}$ has better fitness, the worst frog $X_{w}$ will be replaced. Otherwise, the calculations in (1) are repeated with replacement of $X_{b}$ by $X_{g}$. If no improvement occurs in this case, a new solution is randomly generated within the feasible space to replace the worst frog $X_{w}$. Then, the calculations continue for a specific number of iterations. After a prespecified number of memetic evolutionary steps within each memeplex, to ensure global exploration, ideas passed within memeplexes are combined in the shuffling process. All the frogs are resorted, and the population is redivided into $m$ memeplexes. The concurrently implemented local search and global shuffling continue alternatively until predefined convergence criteria are satisfied.

\section{Charging Optimization Model for EV Battery Swap Station}

Large-scale charging behavior of the EV will have a serious impact on the grid. Coordinated charging of the EV battery swap station can reduce the difference between the growing load peak and off-peak and save the costs of grid operation.

3.1. Charging Power Model of the EV Battery Charger. In the EV battery swap station, the typical strategy for battery charging is a two-stage method. The first stage has a constant current and limited pressure and the second stage has a constant pressure and limited current. Charging load power can be expressed as

$$
P(t)= \begin{cases}I_{\max } U(t), & 0 \leq t \leq T_{C} \\ U_{\max } I(t), & T_{C}<t \leq T_{F}\end{cases}
$$

where $I_{\max }$ and $U_{\max }$ denote the maximum charging current and voltage, respectively. $T_{C}$ is the duration of charging with a constant current and $T_{F}$ is the total charging duration. These parameters are constants determined by the battery type and charging characteristics.

In the first stage, to facilitate the modeling and analysis, the charging voltage is treated as the linear representation of $U_{\max }$. And in the second stage, the charging current is exponentially declined with time [20]. Then (2) can be rewritten as

$$
P(t)= \begin{cases}I_{\max } U_{\max }\left[(1-k) \frac{t}{T_{C}}+k\right], & 0 \leq t \leq T_{C}, \\ U_{\max } I_{\max } e^{-\alpha\left(t-T_{C}\right)}, & T_{C}<t \leq T_{F},\end{cases}
$$

where $\alpha$ and $k$ are also constants determined by the battery type and charging characteristics.

In order to investigate the optimal control strategy for coordinated charging, the total charging duration $T_{F}$ is divided equally into $M$ sections, and the duration $T_{M}$ of each section can be derived as $T / M$. According to the equal area 
rule, the charging power of each section can be expressed with the average power:

$$
\bar{P}_{j}=\frac{\int_{(j-1) T_{M}}^{j T_{M}} P(t) d t}{T_{M}}, \quad 1 \leq j \leq M .
$$

Then an integer $C$ is defined to satisfy the following expression:

$$
C T_{M} \leq T_{C} \leq(C+1) T_{M} .
$$

Substituting (3) and (5) into (4) yields the multisection charging power model of battery charger:

$$
=\left\{\begin{array}{cc}
\bar{P}_{j} \\
U_{\max } I_{\max }\left[\frac{(1-k)(2 j-1) T_{M}}{2 T_{C}}+k\right] & 0<j \leq C \\
+k\left(T_{C}-C T_{M}\right) & \\
\left.-\frac{e^{\alpha\left(T_{C}-C T_{M}-T_{M}\right)}-1}{\alpha}\right] & C<j \leq C+1 \\
U_{\max } I_{\max } \frac{e^{\alpha\left[T_{C}-(j-1) T_{M}\right]}-e^{\alpha\left(T_{C}-j T_{M}\right)}}{\alpha} & C+1<j \leq M .
\end{array}\right.
$$

Obviously, the accuracy of (6) is relative to $M$. A larger $M$ implies the higher precision of the model with the greater amount of computation, and vice versa.

\subsection{Optimization Objective Function of the EV Battery Charg-} ing Power. According to the "technical guide for electric vehicle battery-swap station" presented by the State Grid Corporation of China (SGCC), a single EV battery charger is used to charge a single battery box. Inside the battery box, the battery pack consists of a plurality of battery cells. The battery mentioned in this paper refers to the battery pack. There are two types of batteries in the EV battery swap station, namely, the regulated battery connected to the EV battery charger and participating in the grid charging optimization by controlling its charging start time and the full charged reserve battery disconnected to the charger and used when not meeting the demand for swapping battery. Only the regulated-battery is considered in this paper.

In order to investigate the optimal control strategy for coordinated charging of the EV battery swap station, some assumptions are made as follows.

(1) All batteries are of the same type. The initial-charging SOC of each battery is the same and set to $20 \%$, and the endcharging SOC is also the same and set to $100 \%$.

(2) Corresponding to the above multisection charging power model of the battery charger, one optimization period $T_{O}$ is also divided into multisections $\left(M_{\mathrm{O}}\right)$ with each section having the same duration $T_{M}$. And $M_{O}=T_{O} \cdot M / T$. Then in one optimal period $T_{O}$, the number $N_{b}$ of batteries needed for charging can be expressed as

$$
N_{b}=\sum_{j=1}^{M_{O}} b_{j} \quad 1 \leq j \leq M_{O}
$$

where $b_{j}$ is the predicted number of batteries swapped into the station during the $j$ th section. For optimal control, it is necessary to number these batteries needed for optimal charging. From the $b_{0}$ batteries, uncharged and reserved in the swap station at the beginning of the $T_{O}$, to the batteries swapped into the station during the $T_{O}$, the batteries are numbered consecutively until $N_{b}$. The other batteries swapped into the station of the $T_{O}$ are not indexed again and charged during the next optimization period.

(3) For the battery $i, t_{\text {in }}(i)$ and $t_{\text {out }}(i)$ denote its swap-in time and swap-out time, respectively:

$t_{\text {in }}(i)=0$ : battery $i$ is one of the batteries $b_{0}$;

$t_{\text {in }}(i)=t_{j}$ : battery $i$ is swapped in during the $j$ th optimization section;

$t_{\text {out }}(i)=t_{k}$ : battery $i$ is swapped out during the $k$ th optimization section;

$$
t_{\text {out }}(i)=M_{\mathrm{O}}+1 \text { : battery } i \text { is remained in the station. }
$$

The battery can be swapped into and swapped out of the station at any time in one section. To facilitate the optimal control, we suppose that the battery could be charged from the section next to that of swapping in and be swapped out of the station from the section next to that of full charged. Then the start-charging time $T_{S}(i)$ of the battery $i$ is between $t_{\mathrm{in}}(i)+1$ and $t_{\mathrm{out}}(i)-M$ :

$$
t_{\text {in }}(i)+1 \leq T_{S}(i) \leq t_{\text {out }}(i)-M .
$$

To ensure that all batteries are full charged at the end of the $T_{O}$, the swapped-in batteries during the last $M$ sections of the $T_{O}$ are no longer charged. Then the start-charging time $T_{S}\left(N_{b}\right)$ of the battery $N_{b}$ would meet

$$
\begin{gathered}
b_{0}+\sum_{j=1}^{T_{S}\left(N_{b}\right)-1} b_{j} \leq N_{b} \leq b_{0}+\sum_{j=1}^{T_{S}\left(N_{b}\right)} b_{j}, \\
T_{S}\left(N_{b}\right) \leq M_{O}-M .
\end{gathered}
$$

If there are $i_{f 0}$ full charged batteries at the beginning of the $T_{O}$ and all swapped out in the $t_{f}$ section, the new full charged batteries would be swapped out from the $t_{f}$ section. Then

$$
\sum_{j=1}^{t_{f}-1} b_{j} \leq i_{f 0} \leq \sum_{j=1}^{t_{f}} b_{j}, \quad t_{f} \geq M+1 .
$$

(4) The battery is the core of EV and quite expensive. Many times of start and stop charging would greatly reduce its life, in which case the battery charging continuity constraint is introduced. 
Based on assumptions (1) and (4), the charging duration of all batteries is the same. In this paper, the minimum variance of the load profile including the charging loads generated by the EV battery swap station is the optimization objective and is expressed as

$$
\begin{aligned}
& \min F=\frac{\min \left[\sum_{j=1}^{M_{O}}\left(P_{d, j}+\sum_{i=1}^{N} C_{i, j} P_{i, j}-P_{\mathrm{avr}}\right)^{2}\right]}{M_{O}} \\
& \text { S.T. }\left\{\begin{array}{l}
b_{0}+\sum_{j=1}^{T_{S}\left(N_{b}\right)-1} b_{j} \leq N_{b} \leq b_{0}+\sum_{j=1}^{T_{S}\left(N_{b}\right)} b_{j} \\
T_{S}\left(N_{b}\right) \leq M_{O}-M \\
t_{f}-1 \\
\sum_{j=1} b_{j} \leq i_{f 0} \leq \sum_{j=1}^{t_{f}} b_{j} \\
t_{f} \geq M+1 \\
t_{\text {in }}(i)+1 \leq T_{S}(i) \leq t_{\text {out }}(i)-M,
\end{array}\right.
\end{aligned}
$$

where

$$
P_{\mathrm{avr}}=\frac{\sum_{j=1}^{M_{O}}\left(P_{d, j}+\sum_{i=1}^{N} C_{i, j} P_{i, j}\right)}{M_{O}},
$$

$P_{d, j}$ : the predicted daily load power of the $j$ th section excluding the charging load generated by EV swap station;

$N$ : the total number of EV battery chargers;

$C_{i, j}$ : the charging decision variable of the $i$ th battery during the $j$ th section: " 1 " means charging and " 0 " is discharging. Based on assumption (4),

$$
C_{i, j}= \begin{cases}1 & T_{S}(i) \leq j \leq T_{S}(i)+M-1 \\ 0 & \text { else; }\end{cases}
$$

$P_{i, j}$ : the charging load of the $i$ th battery during the $j$ th section.

\section{ISFLA-Based Optimal Control Strategy for Coordinated Charging}

There are $N$ chargers controlled by the control center of the EV battery swap station. From (11), the optimal control of the battery coordinated charging is a multivariable, nonlinear, and discrete integer optimization problem. Using the reflecting method to deal with the boundary constraint, an improved shuffled frog leaping algorithm (ISFLA) is proposed to achieve the optimal control strategy for coordinated charging of EV battery swap station.

4.1. Definition of Frog Position. In this paper, the position of the virtual frog consists of the start-charging time of every battery needed for coordinated charging, so the dimension of solution space is equal to the number $N_{b}$ of the regulated battery. The $i$ th frog can be expressed as

$$
X_{i}=\left(T_{i s}(1), T_{i s}(2), \ldots, T_{i s}\left(N_{b}\right)\right) .
$$

Based on the assumptions,

$$
T_{i s}(h) \in \begin{cases}{\left[1, M_{O}-M\right]} & 1 \leq h \leq b_{0}, \\ {\left[K+2, M_{O}-M\right]} & \sum_{j=0}^{K} b_{j}+1 \leq h \leq \sum_{j=0}^{K+1} b_{j},\end{cases}
$$

where $0 \leq K \leq M_{O}-M-2$.

4.2. Inner-Memeplex Evolution. $T_{\text {is }}$ is an integer, and the position of the frog with the worst fitness is adjusted as the following modified rule:

$$
\begin{aligned}
& d_{j}=\operatorname{rand}(0,1) \times\left(T_{b s}(j)-T_{w s}(j)\right), \quad 0 \leq j \leq N_{b}, \\
& T_{w \_ \text {new }}(j)=T_{w s}(j)+\left[d_{j}\right], \quad-d_{j, \max } \leq d_{j} \leq d_{j, \max },
\end{aligned}
$$

where $\left[d_{j}\right]$ means an integer rounded to $d_{j}$.

4.3. Boundary Constraint. Dealing with the boundary constraint may affect the performance of the algorithm. In general, there are three basic approaches, namely, absorbing, reflecting, and damping. Using the reflecting method, when the updating position of the frog after $t$ iterations is beyond a certain boundary, the frog would return within the boundary with the original speed but in the opposite direction. In this paper, the reflecting method is chosen to deal with the boundary constraint and the position evolution of the frog obeys the following rule.

When $d_{j}>0$,

$$
\begin{aligned}
& T_{w s_{\text {new }}}(j) \\
& = \begin{cases}T_{w s}(j)-\left[d_{j}\right] & T_{w s}(j)=M_{O}-M, \\
\min \left(T_{w s}(j)+\left[d_{j}\right], M_{O}-M\right) & T_{w s}(j)<M_{O}-M ;\end{cases}
\end{aligned}
$$

when $d_{j}<0$,

$$
\begin{aligned}
& T_{w s_{\text {new }}}(j) \\
& = \begin{cases}T_{w s}(j)-\left[d_{j}\right] & T_{w s}(j)=t_{\text {in }}(j)+1, \\
\max \left(T_{w s}(j)+\left[d_{j}\right], t_{i n}(j)+1\right) & T_{w s}(j)>t_{\text {in }}(j)+1 .\end{cases}
\end{aligned}
$$

4.4. Steps of the ISFLA. The overall process of the ISFLA can be described in the following main steps.

Step 0 (initialize the parameters). Set the parameters of $p, n$, ite $m$, and ite $p$. $p$ is number of frogs in the whole population. $n$ is the number of frogs in each memeplex. ite_m is the maximum number of iterations for each memeplex. ite $p$ is the maximum number of shuffling iterations for whole population. Therefore, $m=p / n$ is the number of memeplexes. 
TABLE 1: Basic parameters of battery.

\begin{tabular}{lcccc}
\hline Type & $U_{\max }(\mathrm{V})$ & $I_{\max }(\mathrm{A})$ & $\begin{array}{c}\text { Constant } \\
\text { current charging } \\
\text { duration (min) }\end{array}$ & $\begin{array}{c}\text { Constant } \\
\text { voltage charging } \\
\text { duration (min) }\end{array}$ \\
\hline Li-Ion & 58.8 & 120 & 90 & 150 \\
\hline
\end{tabular}

Step 1 (initialize the population). The initial population is formed by $p$ randomly generated frogs: $X_{1}, X_{2}, \ldots, X_{p}$. Compute the fitness value $F\left(X_{i}\right)$ according to (11) for each frog $X_{i}$.

Step 2 (shuffle the frogs). Firstly sort the $p$ frogs in order of decreasing fitness, and then partition all the $p$ frogs into $m$ memeplexes. The first frog goes to the first memplex, the second frog goes to the second memeplex, the $m$ th frog goes to the $m$ th memeplex, the $(m+1)$ th frog goes back to the first memeplex, and at last the $p$ th frog goes to the $m$ th memeplex.

Step 3 (memetic evolution). Repeat the following operations for ite $m$ times: firstly find the worst frog $X_{w}$ and the best frog $X_{b}$ in each memeplex, then update $X_{w}$ according to (16)(18), and yield $X_{w \text { new }}$. If $X_{w_{\text {nnew }}}$ has a better fitness, replace $X_{w}$ by $X_{w \_n e w}$. Otherwise, repeat the updated strategy with $X_{g}$ replacing $X_{b}$. If this still cannot produce a better solution, replace the $X_{w}$ by a randomly generated frog.

Step 4 (check convergence). If the convergence criteria are met, stop and output the best frog $X_{q}$ in the whole population. Otherwise, return to Step 2.

In this ISFLA, the convergence criteria are defined as the maximum number of shuffling iterations for whole population (ite_p).

\section{Numerical Simulation}

In this paper, the optimization objective is one area's daily load profile which includes the charging loads generated by the EV battery swap station. The optimization period $T_{O}$ is set to a day and divided into 24 sections with the duration $T_{M}$ of one hour.

5.1. Parameters of EV Battery. In the EV market, there are different battery types such as NiMH, Lead Acid, and Li-Ion. And the market share of Li-Ion with its technical advantages has increased annually. The "E6 pioneer" EV developed by BYD Co., Ltd., has been configured a Li-Ion battery with the energy density of $100 \mathrm{~W} \cdot \mathrm{h}$ per kilogram (i.e., a battery of $600 \mathrm{~kg}$ can store power of $60 \mathrm{~kW} \cdot \mathrm{h}$ for each charging). Its parameters are shown in Table 1.

In consideration of the charging continuity constraint and based on the battery parameters, the average charging power of each section in the multisection charging model can be calculated and shown in Table 2.

5.2. Area Daily Load. A certain area daily load power before regulation can be predicted and shown in Table 3 .
TABLE 2: Average charging power of each section.

\begin{tabular}{lcccc}
\hline Section & 1 & 2 & 3 & 4 \\
\hline Charging power $(\mathrm{kW})$ & 6.45 & 6.18 & 2.52 & 0.85 \\
\hline
\end{tabular}

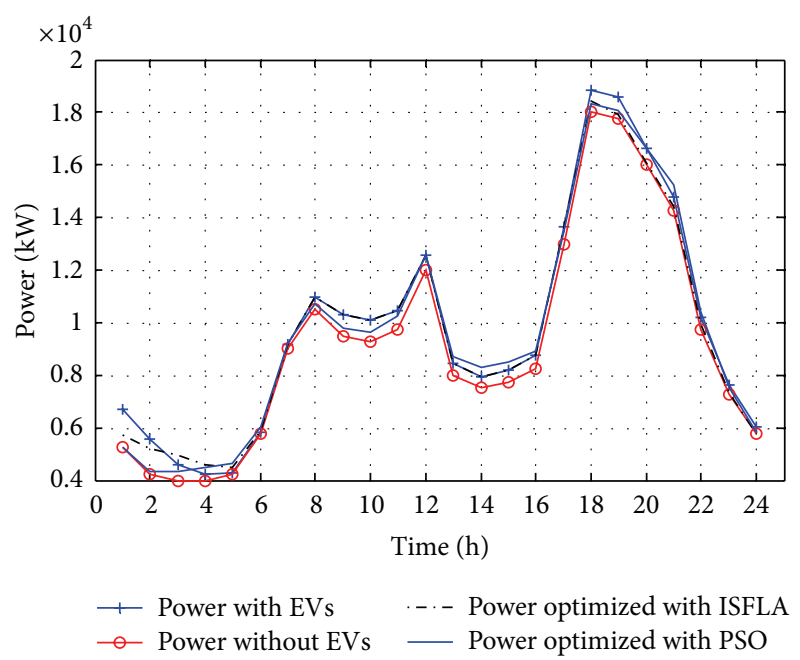

FIGURE 1: Area load profile under different conditions.

5.3. Parameters of the EV Battery Swap Station. At the beginning of one optimization period, there are 600 batteries in the swap station, including 350 full charged batteries, 200 unfull changed batteries, and 50 reserved batteries. All batteries except the reserved batteries participate in the optimal control of coordinated charging. By predicting the demand of EV owners for swapping batteries during each optimization section, the number $b_{j}$ can be derived and shown in Table 4 .

5.4. Simulation of ISFLA. The main parameters of ISFLA are set and shown in Table 5. Based on ISFLA and PSO, the area load profiles are compared and shown in Figure 1.

In Figure 1, ISFLA and PSO can lower the load peakvalley difference and smooth the load profile. But the convergence characteristics of two algorithms in the numerical simulation differ greatly and are shown in Figure 2.

Clearly, the convergence rate of ISFLA is much faster than that of PSO and the minimum variance of the load profile generated by ISFLA is less than that generated by PSO. Under different conditions, the variances of the load profile are shown in Table 6.

With ISFLA, the distribution of batteries according to the start-charging time in the optimization period $T_{O}$ is shown in Figure 3.

In Figure 3, during some optimization sections, there is a very small or no difference between the number of startingcharging batteries and that of those swapped into the station. This can explain that the optimized load profile with ISFLA and the load profile with uncoordinated charging almost coincide in some sections in Figure 1.

For a fixed population $p$, the different number $m$ of partitioned memeplexes will affect the convergence rate and the global optimal solution. Some simulations are made of 
TABLE 3: Certain area daily load before regulation.

\begin{tabular}{|c|c|c|c|c|c|c|c|}
\hline Time (h) & Power (MW) & Time $(\mathrm{h})$ & Power (MW) & Time (h) & Power (MW) & Time (h) & Power (MW) \\
\hline 1 & 5.25 & 7 & 9 & 13 & 8 & 19 & 17.75 \\
\hline 2 & 4.25 & 8 & 10.5 & 14 & 7.5 & 20 & 16 \\
\hline 3 & 4 & 9 & 9.5 & 15 & 7.75 & 21 & 14.25 \\
\hline 4 & 4 & 10 & 9.25 & 16 & 8.25 & 22 & 9.75 \\
\hline 5 & 4.25 & 11 & 9.75 & 17 & 13 & 23 & 7.25 \\
\hline 6 & 5.75 & 12 & 12 & 18 & 18 & 24 & 5.75 \\
\hline
\end{tabular}

TABLE 4: Predicted number of batteries swapped in during each optimization section.

\begin{tabular}{lccccccccccccc}
\hline Time (h) & 1 & 2 & 3 & 4 & 5 & 6 & 7 & 8 & 9 & 10 & 11 & 12 \\
\hline Number & 3 & 3 & 1 & 2 & 10 & 18 & 53 & 68 & 46 & 31 & 17 & 24 \\
\hline Time $(\mathrm{h})$ & 13 & 14 & 15 & 16 & 17 & 18 & 19 & 20 & 21 & 22 & 23 & 24 \\
\hline Number & 30 & 30 & 37 & 50 & 67 & 42 & 28 & 28 & 25 & 18 & 13 & 6 \\
\hline
\end{tabular}

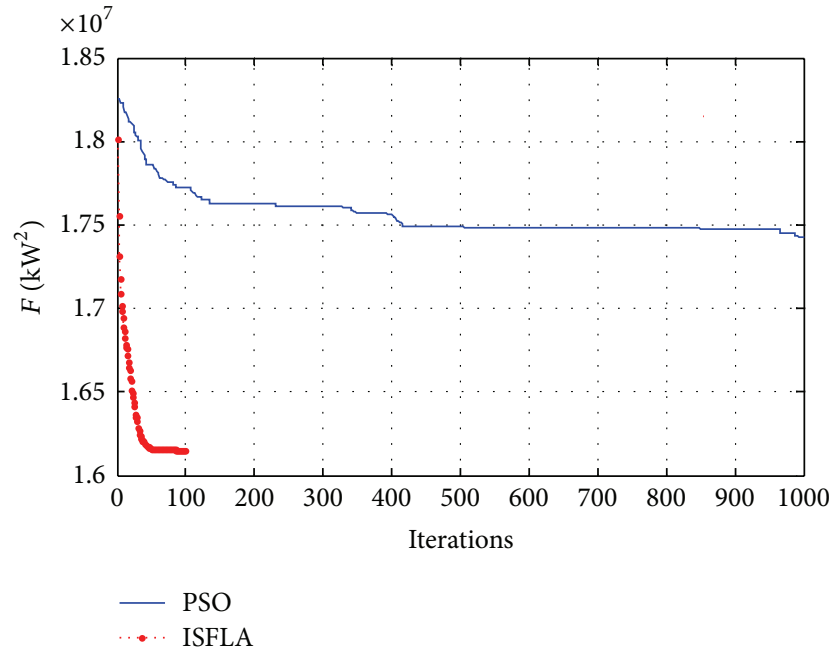

FIGURE 2: Convergence characteristics of ISFA and PSO.

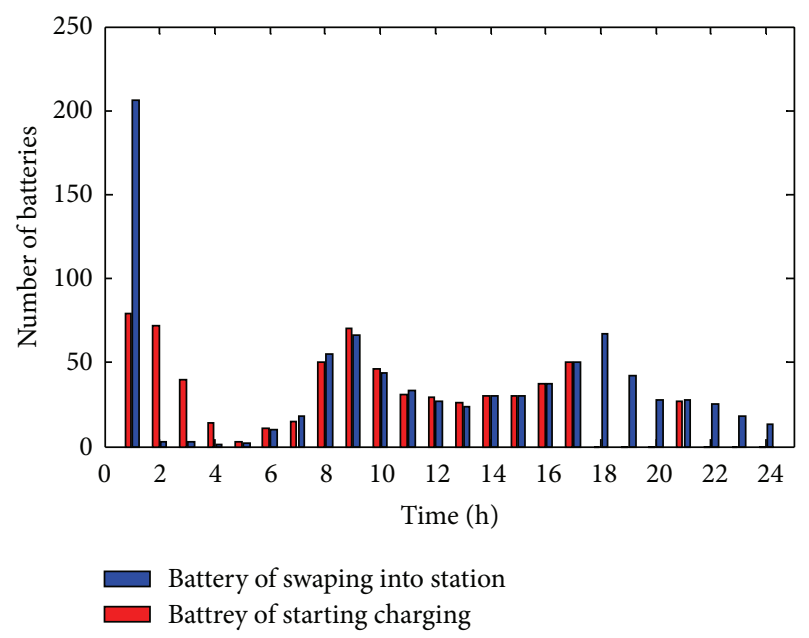

FIGURE 3: Distribution of batteries starting charging.
TABLE 5: Parameters of ISFLA.

\begin{tabular}{lccccc}
\hline Parameter & $p$ & $m$ & $n$ & ite_m & ite_ $p$ \\
\hline Value & 50 & 10 & 5 & 5 & 100 \\
\hline
\end{tabular}

TABLE 6: Variances under different conditions.

\begin{tabular}{lccc}
\hline Condition & $\begin{array}{c}\text { Uncoordinated } \\
\text { charging }\end{array}$ & \multicolumn{2}{c}{ Coordinated charging } \\
Variance $\left(\mathrm{kW}^{2}\right)$ & 1.836 & 1.615 & PSO \\
Result & - & Declining $12.04 \%$ & Declining $5.40 \%$ \\
\hline
\end{tabular}

TABLE 7: Global convergence value.

\begin{tabular}{lcccc}
\hline Memeplex & 5 & 10 & 20 & 40 \\
\hline$F\left(\mathrm{~kW}^{2}\right) \times 10^{7}$ & 1.616 & 1.615 & 1.6139 & 1.6137 \\
\hline
\end{tabular}

ISFLA with the parameters of a fixed $p$ (200) and different $m$ and the results are shown in Figure 4 and Table 7.

In Figure 4 and Table 7, the greater the number of memeplex is, the faster the algorithm converges with a higher convergence performance. However, the relationship between them is not linear, and how to choose an appropriate memeplex considering the convergence rate and global convergence value is a main goal of our further work.

\section{Conclusion}

As a main way of energy supply to EVs, the optimal control strategy for coordinated charging of the swap station is very important in smoothing the load profile. Based on the characteristics of the EV battery swap station, a multisection charging power model of battery is presented and an ISFLA in reflecting method to deal with the boundary constraint is proposed to achieve coordinated charging of batteries. In numerical simulations, the comparison of PSO and ISFLA is made, and the results show that the presented ISFLA can effectively lower the peak-valley difference and smooth 


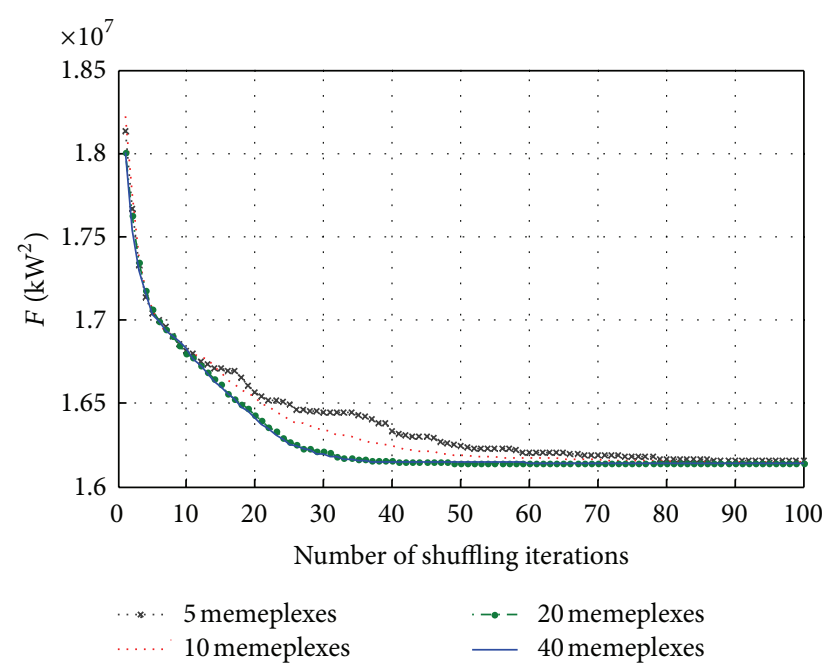

FIGURE 4: Iterations of ISFLA with different memeplexes.

the load profile with the faster convergence rate and higher convergence precision.

\section{Acknowledgments}

This work was supported by the National High-Tech Research \& Development Program of China (“863” Program) (Grant no. 2012AA050210), the National Natural Science Foundation of China (Grant no. 51177011), and Provincial Science and Technology Supporting Program (Grant no. BE2011174).

\section{References}

[1] Q. Q. Chen and L. Q. Sun, "Present statue and future trends of electric vehicles," Science \& Technology Review, vol. 23, no. 4, pp. 24-28, 2011 (Chinese).

[2] K. Schneider, C. Gerkensmeyer, M. Kintner-Meyer, and R. Fletcher, "Impact assessment of plug-in hybrid vehicles on pacific northwest distribution systems," in Proceedings of the IEEE Power and Energy Society General Meeting: Conversion and Delivery of Electrical Energy in the 21st Century, pp. 1-6, July 2008.

[3] S. Acha, T. C. Green, and N. Shah, "Impacts of plug-in hybrid vehicles and combined heat and power technologies on electric and gas distribution network losses," in Proceedings of the 1st IEEE-PES/IAS Conference on Sustainable Alternative Energy (SAE '09), pp. 1-7, September 2009.

[4] W. Su and M.-Y. Chow, "Performance evaluation of an EDAbased large-scale plug-in hybrid electric vehicle charging algorithm," IEEE Transactions on Smart Grid, vol. 3, no. 1, pp. 308315, 2012.

[5] K. Clement-Nyns, E. Haesen, and J. Driesen, "The impact of charging plug-in hybrid electric vehicles on a residential distribution grid," IEEE Transactions on Power Systems, vol. 25, no. 1, pp. 371-380, 2010.

[6] F. Lanati, M. Benini, and A. Gelmini, "Impact of the penetration of electric vehicles on the Italian power system: a 2030 scenario," in Proceedings of the IEEE PES General Meeting: The Electrification of Transportation and the Grid of the Future, pp. 1-8, July 2011.
[7] R. J. Bessa, M. A. Matos, F. J. Soares, and J. A. P. Lopes, "Optimized bidding of a EV aggregation agent in the electricity market," IEEE Transactions on Smart Grid, vol. 3, no. 1, pp. 443452, 2012.

[8] W. Q. Tian, J. H. He, J. C. Jiang, L. Y. Niu, and X. I. Wand, "Research on dispatching strategy for coordinated charging of electric vehicle battery swapping station," Power System Protection and Control, vol. 40, no. 21, pp. 114-119, 2012.

[9] M. Eusuff, K. Lansey, and F. Pasha, "Shuffled frog-leaping algorithm: a memetic meta-heuristic for discrete optimization," Engineering Optimization, vol. 38, no. 2, pp. 129-154, 2006.

[10] M. M. Eusuff and K. E. Lansey, "Optimization of water distribution network design using the shuffled frog leaping algorithm," Journal of Water Resources Planning and Management, vol. 129, no. 3, pp. 210-225, 2003.

[11] X. Pan, "The study on the shuffled frog leap algorithm and its application," Advances in Information Sciences and Service Sciences, vol. 4, no. 1, pp. 215-223, 2012.

[12] X.-H. Luo, Y. Yang, and X. Li, "Modified shuffled frog-leaping algorithm to solve traveling salesman problem," Journal on Communications, vol. 30, no. 7, pp. 130-135, 2009 (Chinese).

[13] J.-P. Luo and X. Li, "Improved shuffled frog leaping algorithm for solving TSP," Journal of Shenzhen University Science and Engineering, vol. 27, no. 2, pp. 173-179, 2010.

[14] J. Ebrahimi, S. H. Hosseinian, and G. B. Gharehpetian, "Unit commitment problem solution using shuffled frog leaping algorithm," IEEE Transactions on Power Systems, vol. 26, no. 2, pp. 573-581, 2011.

[15] J. M. Anita and I. J. Raglend, "Solution of unit commitment problem using shuffled frog leaping algorithm," in Proceedings of the International Conference on Computing, Electronics and Electrical Technologies, pp. 109-115, 2012.

[16] K. Selvakumar, R. S. Vignesh, and R. Vijayabalan, "Shuffled Frog Leaping Algorithm for solving profit based unit commitment problem," in Proceedings of the International Conference on Computing, Communication and Applications (ICCCA '12), pp. 1-6, February 2012.

[17] W. Z. Liu, S. Li, Y. Xiang, and X. Y. Tang, "Virtual network embedding based on shuffled frog leaping algorithm in TUNIE," International Journal of Advancements in Computing Technology, vol. 3, no. 11, pp. 402-409, 2011.

[18] X. D. Zhang, Y. Q. Bao, J. Xi, L. Zhao, and C. R. Zou, "Whispered speech emotion recognition based on improved shuffled frog leaping algorithm neural network," Journal of Convergence Information Technology, vol. 7, no. 19, pp. 121-130, 2012.

[19] X. Zhang, Y. Zhang, Y. Shi, L. Zhao, and C. Zou, "Power control algorithm in cognitive radio system based on modified Shuffled Frog Leaping Algorithm," AEU-International Journal of Electronics and Communications, vol. 66, no. 6, pp. 448-454, 2012.

[20] C. Chen, R. Xie, and X. N. He, "Research of multi-stage charging method for on-board lithium battery of electric vehicles," Journal of Mechanical \& Electrical Engineering, vol. 28, no. 7, pp. 887-890, 2011 (Chinese). 


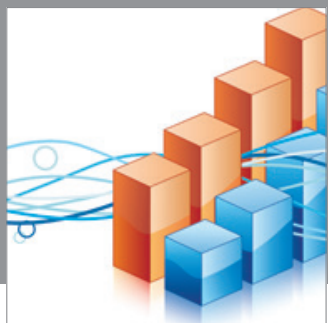

Advances in

Operations Research

mansans

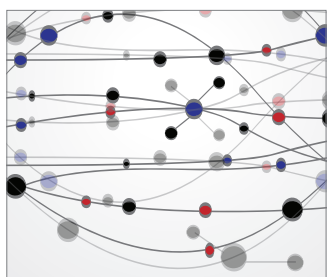

The Scientific World Journal
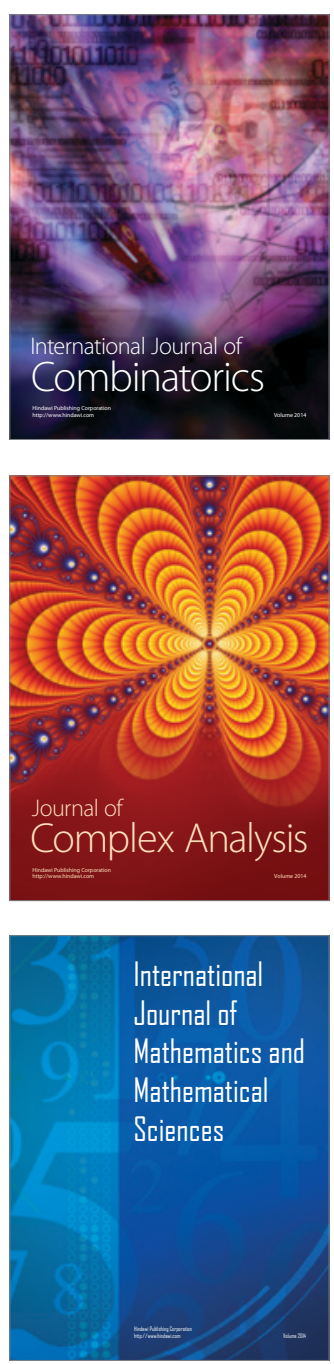
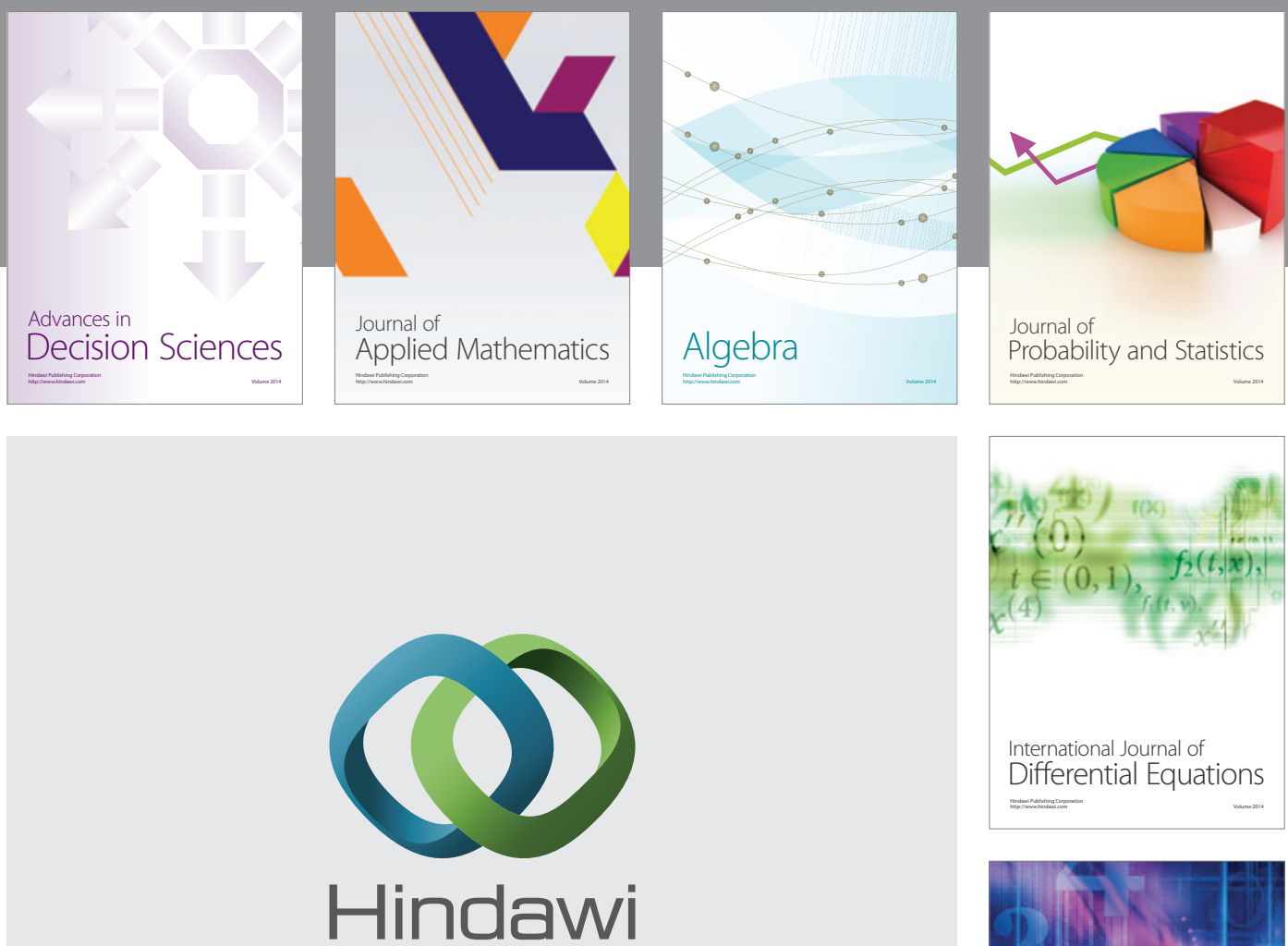

Submit your manuscripts at http://www.hindawi.com
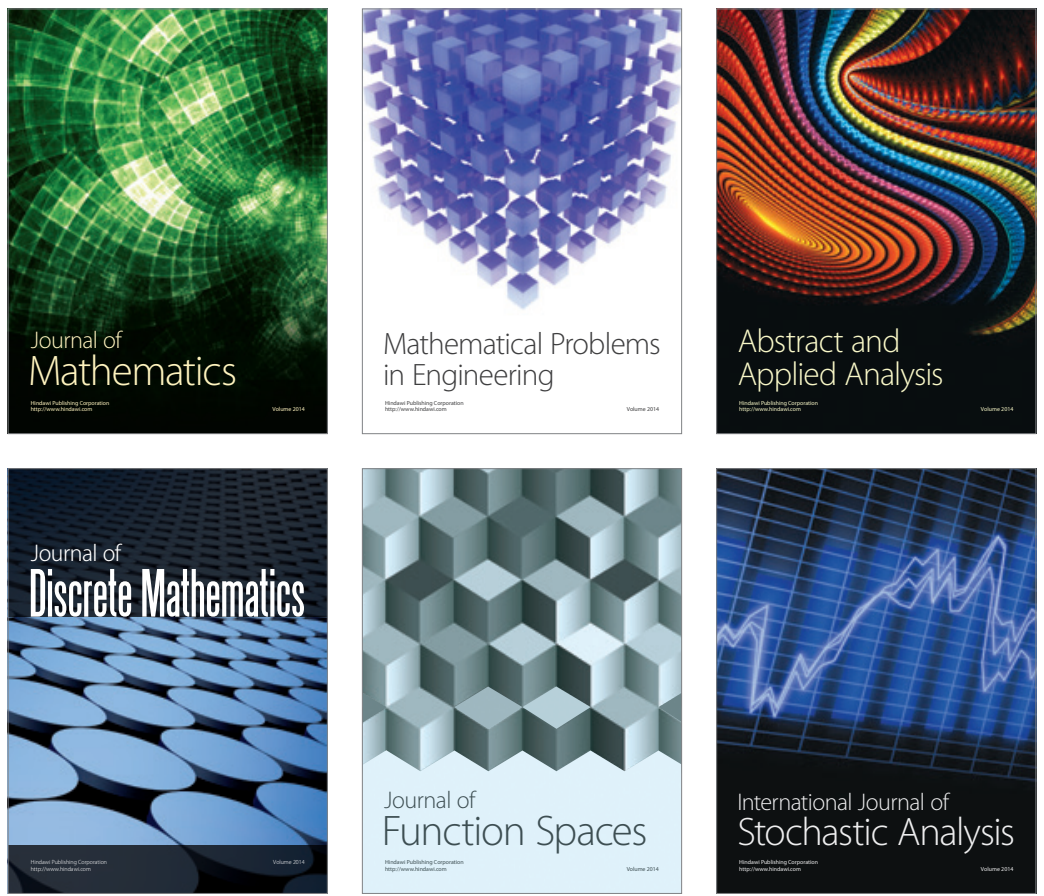

Journal of

Function Spaces

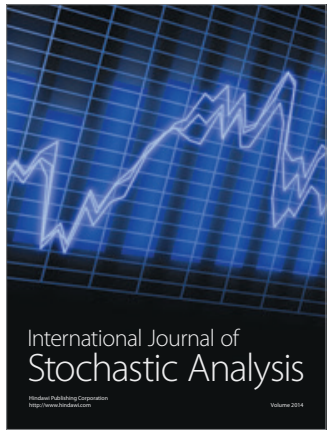

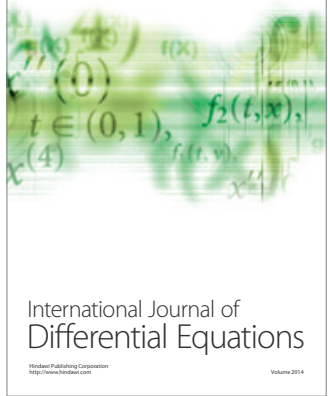
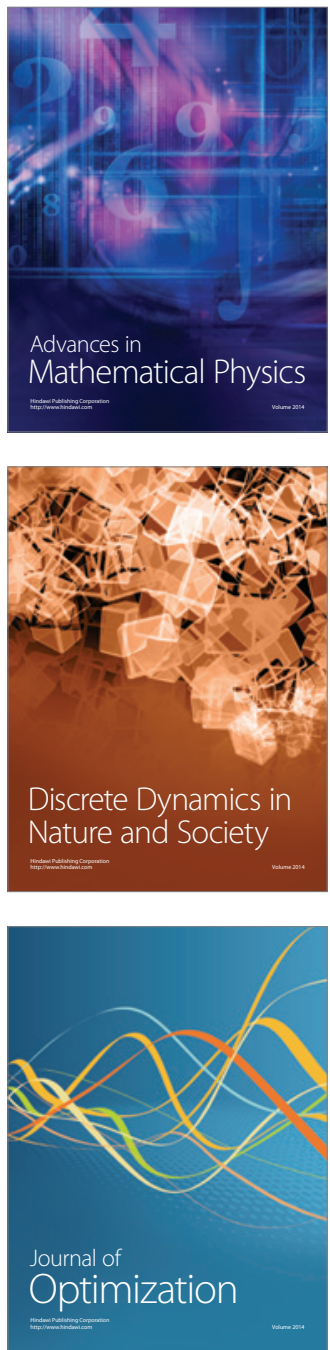